\title{
Study on the fractal dimension of the diameter distribution of electrospun nanofibres non-woven fabrics and the relationship between it and with filtration efficiency
}

\author{
CHEN Ying ${ }^{1,2, a}$, QI Lu ${ }^{1, b}$, LIU Yong ${ }^{1}$, ZHANG Lei ${ }^{1}$, ZHU Ridong ${ }^{3}$, CHEN \\ Rudong ${ }^{3, *}$
}

1. College of Textiles, Tianjin Polytechnic University, Tianjin, China

aEmail: tjmmt@163.com; bluqi005@sina.com

2. Higher vocational education department , Tianjin University of Technology and Education, Tianjin, China, 300222

3. Departmant of Mathematics, Tianjin Polytechnic University, Tianjin, China, 300387

*The corresponding author: CHEN Rudong, Emai: chenrd@tjpu. edu. cn

Key words: electrospun nanofibres non-woven fabrics; G-P algorithm; image thinning; the diameter distribution; correlation fractal dimension.

Abstract: In this paper, samples are first designed and made based on the orthogonal experiment law. Afterward, image gray processing and image binarization processing are performed on the electrospun nanofibres non-woven fabrics image that is captured by the electron microscope through digital picture processing technique, and then is refined with fiber structure through the image thinning algorithm and the diameter distribution of the simple can be obtained through calculating the average diameter of the fabric distributed in various position. Subsequently, the mathematical treatment is performed to the sample's diameter distribution. G-P algorithm is used to calculate the correlation fractal dimension of the diameter distribution. After acquired with fractal dimension, the mapping relation between the fractal dimension and physical properties of filtration efficiency can be verified through the analysis of numerical relation between the fractal feature of electrospun nanofiber nonwoven fabric and the properties of filtration efficiency, which furthermore provides another scale to the evaluation of the electrostatic spinning nano fiber non-woven cloth.

\section{Introduction and Preliminary}

With the rapid development of nano technology, the more and more attention is obtained from the people on the electrospun nanofibres non-woven fabrics, especially for its unique property. However, as its structure is very complex and irregular, the use of fractal theory provides a predictable research direction ${ }^{[1-3]}$. In this paper, the electrospun nanofibres non-woven fabrics image that is captured by the electron microscope through digital picture processing technique is treated through digital image processing technology (Visual C++ and Matlab program), and then we use the method of mathematical morphology to calculate the diameter distribution of electrospun nanofibres and calculate the fractal dimension ${ }^{[6]}$ of electrospun nanofibres according to fractal theory. In comparison with the physical property of corresponding electrospun nanofibres non-woven fabrics, we determine the relationship between fractal dimension and physical property, which also provide the characterization data for study of electrospun nanofibres non-woven fabrics.

Regarding study of non-woven fabrics fractal properties, with study by box dimension method on fractal property of melt-blown nonwoven fabric, we acquire a series of valuable conclusions ${ }^{[1-3]}$. However, as the obvious fiber distribution of electrospun nanofibres non-woven fabrics, it is evidently not appropriate to use the box dimension. To the characteristics of electrospun nanofibres non-woven fabrics, this paper adopts a new dimension, called as the correlation dimension, which is often used to 
research dynamic system. To the electrospun nanofibres non-woven fabrics' chaotic characteristics of fiber texture distribution, by the correlation dimension, we explore the fractal property of electrospun nanofibres non-woven fabrics by correlation dimension and its relevance with its filterability and filter efficiency physical property.

In 1983, Grassberber and Procaccia suggested G-P algorithm to define and calculate the correlation dimension.

G-P algorithm:

Firstly, assuming the sequence $x_{1}, x_{2}, x_{3}, \mathrm{~L}, x_{n}$, we recombinant a vector space of $\mathrm{M}$ dimension with $\mathrm{N}$ vectors.

$$
X=\left[\begin{array}{c}
X_{1} \\
X_{2} \\
\mathrm{M} \\
X_{N}
\end{array}\right]=\left[\begin{array}{cccc}
x_{1} & x_{1+\tau} & \mathrm{L} & x_{1+(M-1) \tau} \\
x_{2} & x_{2+\tau} & \mathrm{L} & x_{2+(M-1) \tau} \\
\mathrm{M} & \mathrm{M} & & \mathrm{M} \\
x_{N} & x_{N+\tau} & \mathrm{L} & x_{N+(M-1) \tau}
\end{array}\right]
$$

Secondly, to calculate the correlation integral $C_{M}(\delta)$

$$
C_{M}(\boldsymbol{\delta})=\frac{1}{N(N-1)} \sum_{\substack{0 \leq i \leq N, 0 \leq j \leq N \\ i \neq j}}\left[H\left(\boldsymbol{\delta}-\left\|X_{i}-X_{j}\right\|\right)\right]
$$

Here $\left\|X_{i}-X_{j}\right\| \sqrt{\sum_{k=0}^{M-1}\left(x_{i+k \tau}-x_{j+k \tau}\right)^{2}}$

$H(x)$ is Heaviside step function, that

$$
H(x)= \begin{cases}0, & x \leq 0 \\ 1, & x>0\end{cases}
$$

By the definition of $C_{M}(\delta)$, if $\delta>\max \left\{\left\|X_{i}-X_{j}\right\|\right\}$, all points fall over on $\delta$ domain, that is

$$
C_{M}(\delta)=\frac{N(N-1)}{N(N-1)}=1
$$

when $\delta<\min \left\{\left\|X_{i}-X_{j}\right\|\right\}$, all points fall over out of $\delta$ domain, that is

$$
C_{M}(\delta)=\frac{0}{N(N-1)}=0
$$

So $C_{M}(\delta)$ is a cumulative distribution function, which reflects the probability of distance between the two points therefore

$$
C_{M}(\delta)=\left(\frac{\delta}{\delta_{\max }}\right)^{D(M, \delta)}, \delta \leq \delta_{\max }
$$

For certain an appropriate range of $\delta$, dimension of dynamic system and cumulative distribution functions should meet the logarithm linear relationship, hence for that smaller than $\delta_{1}$ and $\delta_{2}$, then as: 
On both sides of the logarithm,

$$
\frac{C_{M}\left(\delta_{1}\right)}{C_{M}\left(\delta_{2}\right)}=\frac{\left(\frac{\delta_{1}}{\delta_{\max }}\right)^{D_{2}\left(M, \delta_{1}\right)}}{\left(\frac{\delta_{2}}{\delta_{\max }}\right)^{D_{2}\left(M, \delta_{2}\right)}}
$$

$$
\ln C_{M}\left(\delta_{1}\right)-\ln C_{M}\left(\delta_{2}\right)=D_{2}\left(M, \delta_{1}\right) \ln \frac{\delta_{1}}{\delta_{\max }}-D_{2}\left(M, \delta_{2}\right) \ln \frac{\delta_{2}}{\delta_{\max }}
$$

When $\left|\delta_{1}-\delta_{2}\right|$ is enough small, there is

$$
D_{2}\left(M, \delta_{1}\right) \approx D_{2}\left(M, \delta_{2}\right)
$$

As a result,

$$
D_{2}(M, \delta)=\frac{\ln C_{M}\left(\delta_{1}\right)-\ln C_{M}\left(\delta_{2}\right)}{\ln \delta_{1}-\ln \delta_{2}}
$$

It follows that,

$$
D_{2}(M, \delta)=\frac{d \ln C_{M}(\delta)}{d \ln \delta}
$$

Therefore $^{D_{2}(M, \delta)}$ is the slope of double logarithm curve $\ln C_{M}(\delta)-\ln \delta$, and we can get the slope of the curve by fitting, thereby estimate the value of $D_{2}(M, \delta)$.

In the process of $D_{2}(M, \delta)$ calculation, it is very important to choose an appropriate value of $\mathrm{M}$. Usually, we adopt the saturated correlation dimension method to determine the value of M, namely that is choose different value of $\mathrm{M}$ from small to large. When $D_{2}(M, \delta)$ has no large change any more as the increase of value of $M$, it can choose the value of $M$ at this moment as embedding dimension. Then in process of change, we can obtain a series of value, select the most stable part of linear fitting, and get the correlation dimension $D_{2}(M, \delta)$.

Diameter measure: For regular object, its width can be measured by principle of similarity theory.

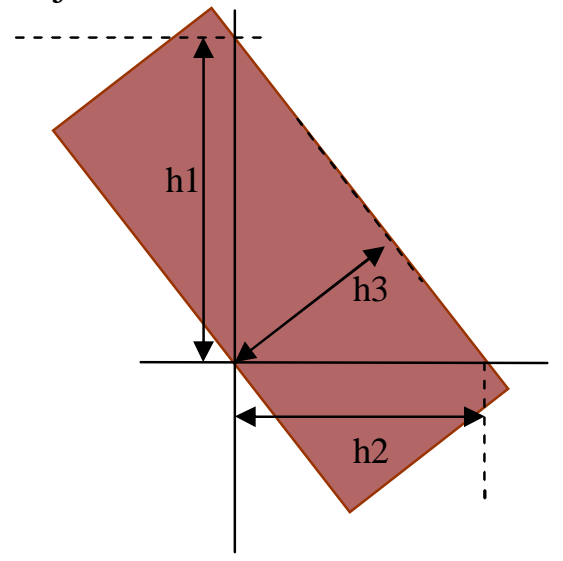

Fig. 1: Width Measurement

As shown in Fig.1, according to the triangle similarity theory, the formula as below:

$$
\frac{h_{2}}{\sqrt{h_{1}^{2}+h_{2}^{2}}}=\frac{h_{3}}{h_{1}}
$$


So its width is:

$$
w=h_{3}=\frac{h_{1} \times h_{2}}{\sqrt{h_{1}^{2}+h_{2}^{2}}}
$$

For the width measurement of similar regular object, the method mentioned above also can be used, but it is more complicate to implementation, especially for so many objects between which even appear overlapped. In this paper, the method we measure similar regular objects width refers to the object thinning rotation covered original image method. This method is theoretically based on the culculas principle.

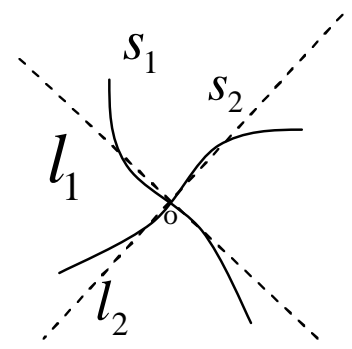

Fig. 2 The graph rotation intersect

As shown in Fig.2, there is 2 curves $s_{1}, s_{2}$, and 2 straight lines $l_{1}, l_{2}$. Curve $s_{2}$ be gotten by $S_{1}$ move round clockwise rotation $90^{\circ}, l_{1}$ is the tangent line $\mathrm{S} 1$ at o, $l_{2}$ is the tangent line $\mathrm{S} 2$ at o. So $l_{1}$ perpendicular to $l_{2}$. By culculas principle, for any $\varepsilon$, exist $\delta>0$, in $O(o, \delta)$, $f\left(l_{2}\right)-f\left(s_{2}\right)<\varepsilon$, here $f(x)$ is the length of curve $x$ in $O(o, \delta)$. So as $\delta$ is full small, $f\left(l_{2}\right) \approx f\left(s_{2}\right)$.

Therefore as object bend is so small, the certain a width of object can be approximately expressed by intersect width center line rotation at this place and object edge. Its implementation effect can be shown in fig.3.

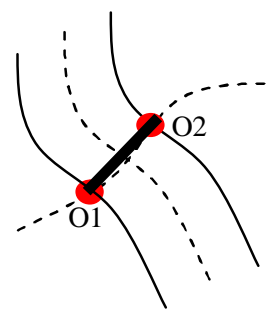

Fig.3 similar width measure

In this paper, because of a great deal of diameter measure, we conduct the measure by the method shown in the figure, which improves the measure efficiency, and can perform the measure by selecting the appropriate position.

Algorithm of Diameter Measure:

Step 1: Choose one base line, which passes through all fibers across the base line from left to right, and summarize the left and the right cross points of each fiber at the same time;

Step 2: Take the center point of left point and right point connection line of each fiber as the center of the square, use the length of connection line as the length of square to make a square, in which, two edges of square should be parallel with the base line.

Step 3: Choose the position corresponding to this thinned image and rotate is at $90^{\circ} \mathrm{ccw}$, the rotated square thinned image is intersected with the corresponding points of original image; 
Step 4: There would be two cross points after intersection, between which the distance refers to the width of fiber; the width value can be calculated through the distance formula.

Algorithm diagram can be shown in Figure 4.

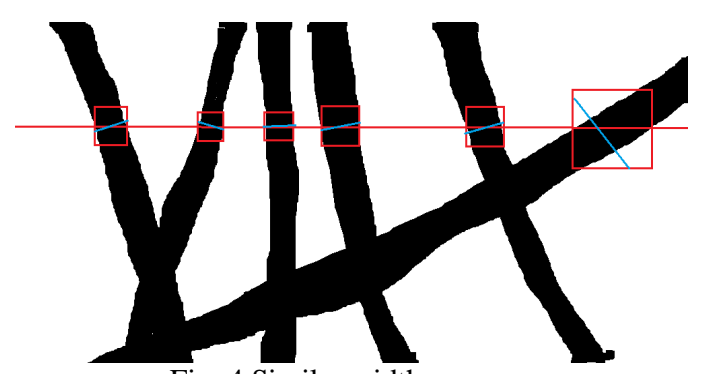

Fig. 4 Similar width measure

\section{Experiments and digital image acquisition}

In order to study the property of electrospun nanofibres non-woven fabrics, we make sample with experimental materials PVA on automatic electrostatic spinning machine DXES-01. There are 5 process parameters to influence electrospun nanofibres non-woven fabrics, there are respectively: time is 90 minutes, Concentration is $14 \%, 15 \%, 16 \%, 17 \%, 18 \%$; distance $(\mathrm{cm})$ is 11, 13, 15, 17, 19; voltage $(\mathrm{kv})$ is $10,15,20,25,26$ and the solution filling $\mathrm{rate}(\mathrm{ml} / \mathrm{h})$ is $0.5,0.7,1,1.2,1.5$. Therefore, in order to obtain more experimental data without too many experiment times, we adopt the mathematic method of orthogonal experimental design. According to the orthogonal table of $\mathrm{L}_{25}\left(5^{6}\right)$ and only four factors related to this problem, we use only its first four columns to design the orthogonal table and measure out each sample's resistance and filter efficiency through the TSI8130 automatic filter tester.

Table 1: The experimental results of permeability and resistance

\begin{tabular}{|c|c|c|c|c|c|c|c|c|c|c|c|}
\hline No. & $\begin{array}{r}\text { Distance } \\
(\mathrm{cm})\end{array}$ & $\begin{array}{r}\text { Voltage } \\
\text { (kv) }\end{array}$ & $\begin{array}{r}\text { Velocity } \\
(\mathrm{ml} / \mathrm{h})\end{array}$ & $\begin{array}{r}\text { Density } \\
\text { (wt\%) }\end{array}$ & $\begin{array}{r}\text { Transmission } \\
(\%)\end{array}$ & No. & $\begin{array}{r}\text { Distance } \\
(\mathrm{cm})\end{array}$ & $\begin{array}{r}\text { Voltage } \\
(\mathrm{kv})\end{array}$ & $\begin{array}{r}\text { Velocity } \\
(\mathrm{ml} / \mathrm{h})\end{array}$ & $\begin{array}{r}\text { Density } \\
\text { (wt } \%)\end{array}$ & $\begin{array}{c}\text { Transmission } \\
(\%)\end{array}$ \\
\hline 1 & 11 & 10 & 0.5 & 14 & 14.3 & 14 & 15 & 25 & 0.5 & 16 & 0.0041 \\
\hline 2 & 11 & 15 & 0.7 & 15 & 0.0053 & 15 & 15 & 26 & 0.7 & 17 & 0.0058 \\
\hline 3 & 11 & 20 & 1 & 16 & 12.14 & 16 & 17 & 10 & 1.2 & 15 & 85.32 \\
\hline 4 & 11 & 25 & 1.2 & 17 & 0.005 & 17 & 17 & 15 & 1.5 & 16 & 15.74 \\
\hline 5 & 11 & 26 & 1.5 & 18 & 0.003 & 18 & 17 & 20 & 0.5 & 17 & 7.0 \\
\hline 6 & 13 & 10 & 0.7 & 16 & 71.68 & 19 & 17 & 25 & 0.7 & 18 & 0.036 \\
\hline 7 & 13 & 15 & 1 & 17 & 1.00 & 20 & 17 & 26 & 1 & 14 & 0.706 \\
\hline 8 & 13 & 20 & 1.2 & 18 & 0.42 & 21 & 19 & 10 & 1.5 & 17 & 85.5 \\
\hline 9 & 13 & 25 & 1.5 & 14 & 57.32 & 22 & 19 & 15 & 0.5 & 18 & 36.32 \\
\hline 10 & 13 & 26 & 0.5 & 15 & 3.89 & 23 & 19 & 20 & 0.7 & 14 & 29.52 \\
\hline 11 & 15 & 10 & 1 & 18 & 77.4 & 24 & 19 & 25 & 1 & 15 & 0.51 \\
\hline 12 & 15 & 15 & 1.2 & 14 & 19.57 & 25 & 19 & 26 & 1.2 & 16 & 0.05 \\
\hline 13 & 15 & 20 & 1.5 & 15 & 9.06 & & & & & & \\
\hline
\end{tabular}

In the paper, the study data refers to the electrospun nanofibres pictures obtained by the desktop scanning electron microscope TM-1000 of Textile College of Tianjin Polytechnic University; Samples' bmp pictures as below: 


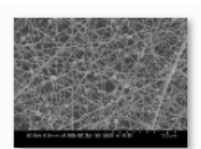

\#1.bmp

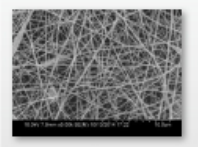

\#7.bmp

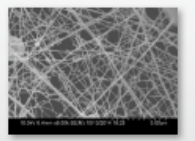

\#13.bmp

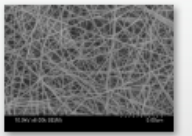

\#19.bmp

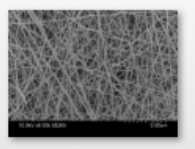

\#25.bmp

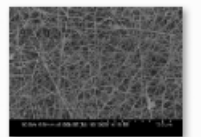

\#2.bmp

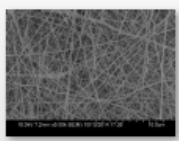

\#8.bmp

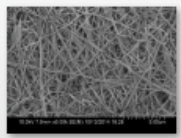

\#14.bmp

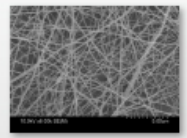

\#20.bmp

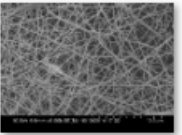

\#3.bmp

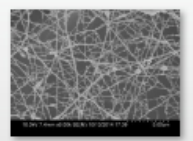

\#9.bmp

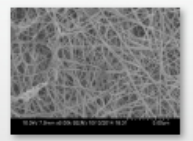

\#15.bmp

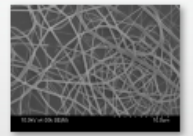

\#21.bmp

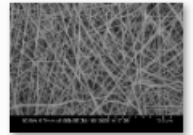

\#4.bmp

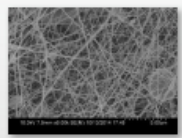

\#10.bmp

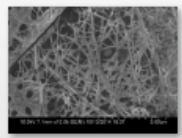

\#16.bmp

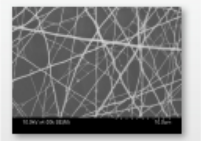

\#22.bmp

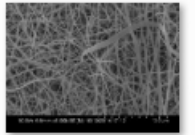

\#5.bmp

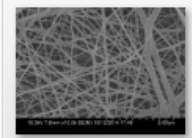

\#11.bmp

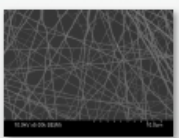

\#17.bmp

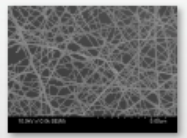

\#23.bmp

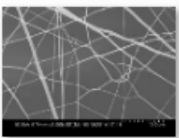

\#6.bmp

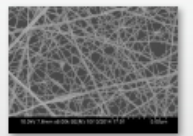

\#12.bmp

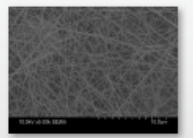

\#18.bmp

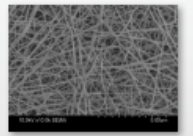

\#24.bmp

Fig. 5 The experimental sample pictures

Digital images is mainly described by pixel, and all digital images are composed of serveral pixels. Pixel in the digital image is generally represented by many values that usually include three primary colors of RGB and four values of transparency. But each pixel of grey image is only indicated by one value, and each pixel value is in the range from 0 to 255 . It is very much conveient to study this image because it has only one value at every point, therefore it is widely used in the digital image processing.

For gray level image, the each pixel just has only one numerical value, and the value is between 0 and 255, therefore it is not convienent to acquire its overall numerial characteristics. According to the characteristics of grey image, we choose the proper threshold value for binaryzation, and then we averagely take 53 different positions in each image according to pixel, measure the diameter of each fiber on the strainge line corrresponding to the pixel, and then calculate the average diameter. With this data, as a basic data of dynamic system, by the G-P algorithm, we can calculate the correlation dimension of all samples.
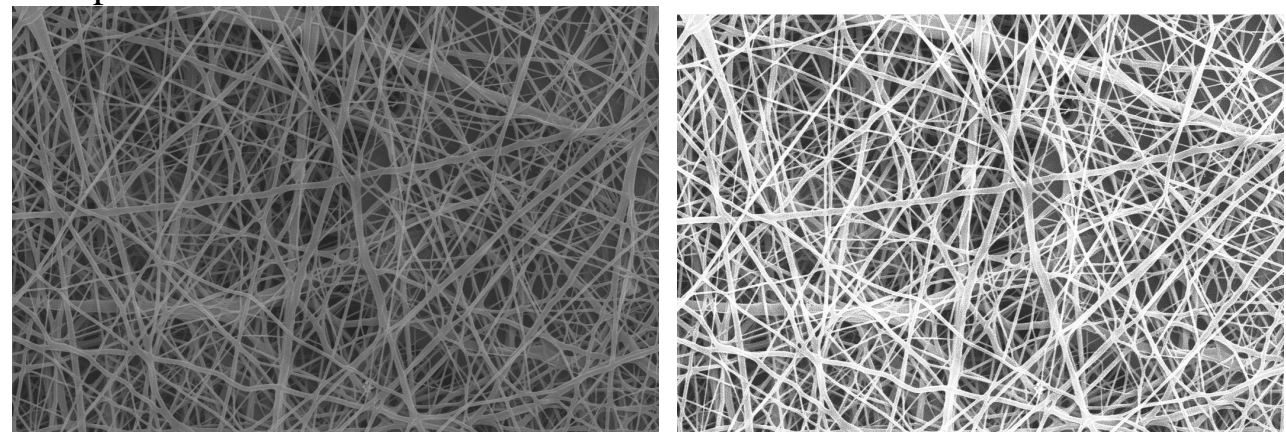

Fig. 6 The original image and the image after histogram equalization 


\section{Digital image thinning, diameter distribution}

Digital image thinning processing would need the knowledge of mathematical morphology [2], Mathematical morphology is based on the set theory, and generally uses the structural elements with some morphological structure, by constantly moving structure element and making a convolution computation to process. The structural element is set as well, the small template for logic judgment; we performed logic judgment by establishing some logical rules.

Now the fiber distribution study is performed on all samples. Take 8\# sample as example, the original image is performed with gray scale image processing, image intensification, image binarization, image denoising, and image thinning, to calculate the diameter distribution with the thinning images and binary images, determine the diameter of each fiber corresponding to the straight line from every 10 pixels from 210 to 760 vertical pixels, and calculate the average value after remove the diameter of the exceptional diameter (For example, the numerical data at the intersection may be relatively large, it would be deleted) it calculates the average value to obtain diameter distribution:

$22.99893,22.48246,20.07918,22.4784,32.56539,25.55126,26.96142,27.35228,28.17349$, $24.45749,28.27732,31.20114,23.12347,23.00741,27.06261,25.19138,28.41876,30.94414$, $33.42561,25.3352$, 28.63172, 23.71931, 25.31117, 19.98035, 21.63209, 27.74743, 31.89576, 25.23462, 21.42972, 30.64564, 17.54497, 24.7021, 26.92871, 20.95673, 25.67032, 26.37167, 23.11428, 20.701, $32.83708,17.09198,25.47207,28.97412$, 22.48886, 25.51947, 25.2574, 27.62504, 24.67204, 18.57486, $26.63576,25.6329,25.63122,19.80336,21.80246,22.96695,19.73825,23.95884$
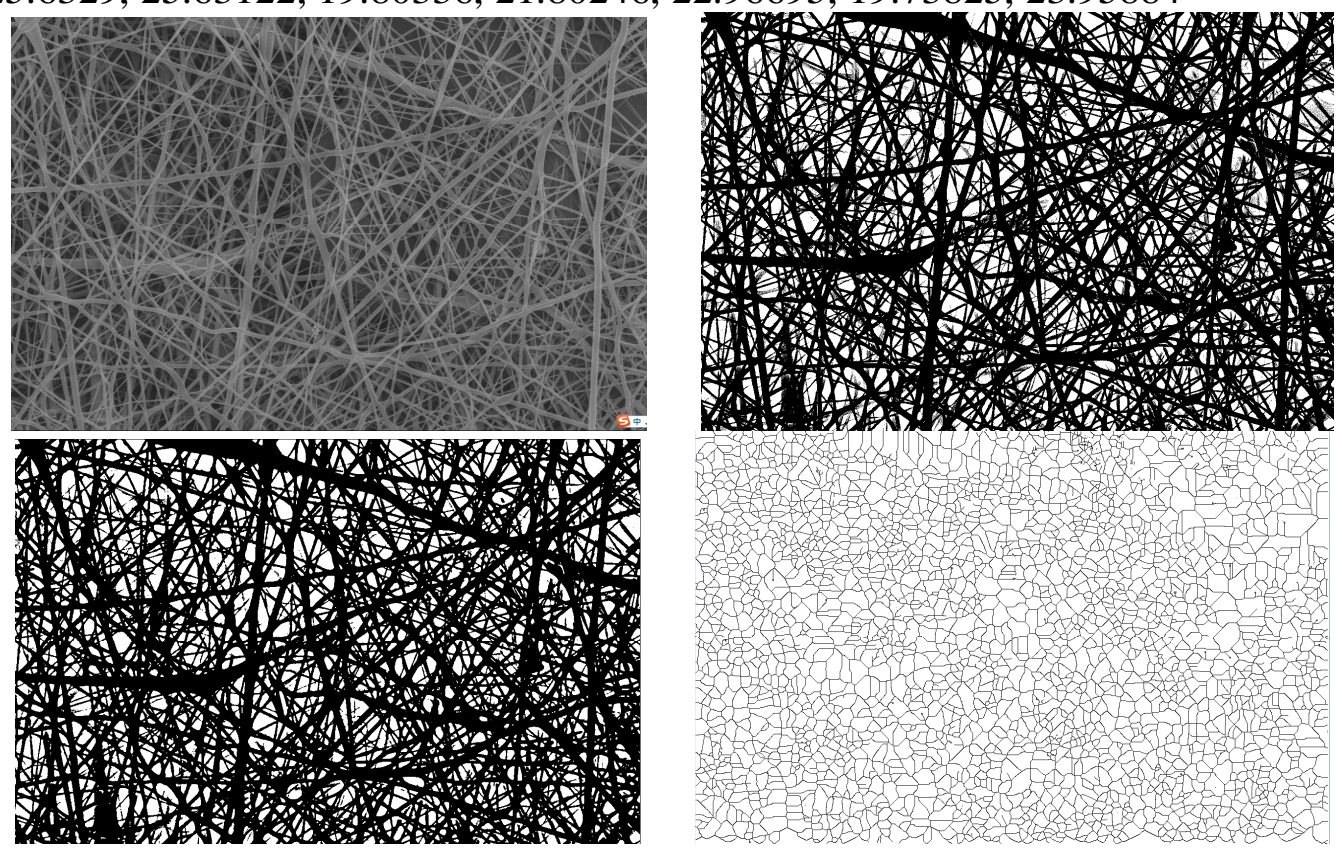

Fig. 7 The whole treatment process image of sample 8\#

For each sample through determination of $\mathrm{VC}++$, it can obtain the fiber diameters corresponding to the different positions as shown in the figure above, which is called as the sample's diameter distribution and is regarded as basic data. After study their fractal dimension according to the indication in the section one, the option of $\mathrm{M}$ value is important in the determination of correlation dimension, therefore we use Matlab to compile the programe, respectively choose $\mathrm{M}=5,10,15,20,25,30,35,40$, and then observe the change of double logarithmic curve, afterward, choose the suited $\mathrm{M}$ value, at this moment, the double logarithmic curves had a relative wide linear range, the sample can be considered to have fractural structure. Therefore, the fractal dimension could be calculated by linear fitting in the range by Matlab. 
Take 8\# as example as well, in Figure 8, each mark number in each picture is the digital double logarithmic curve graph corrresponding to the value of $\mathrm{M}$

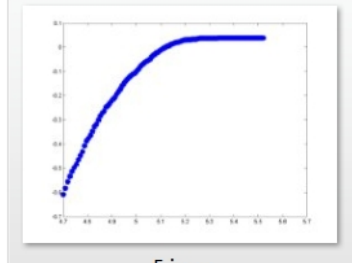

5.jpeg

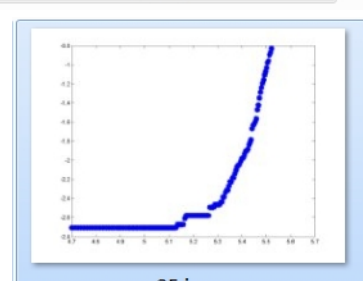

25.jpeg

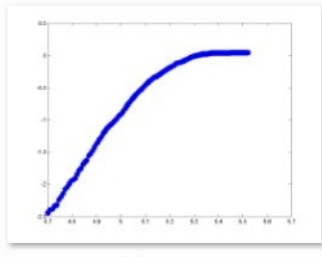

10.jpeg

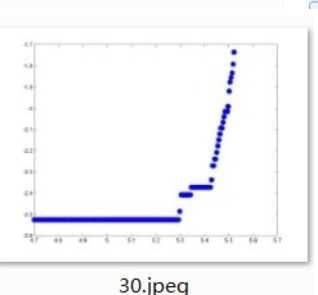

30.jpeg

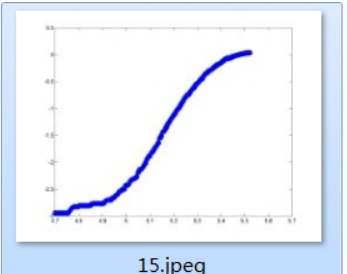

15.jpeg

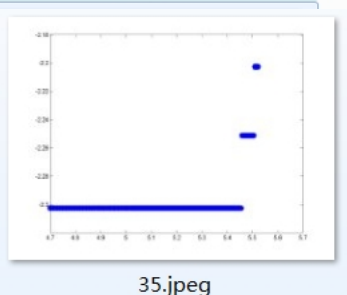

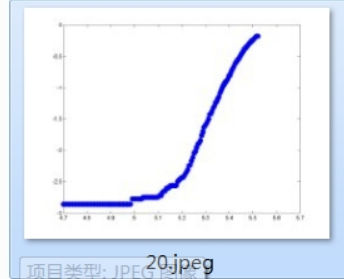

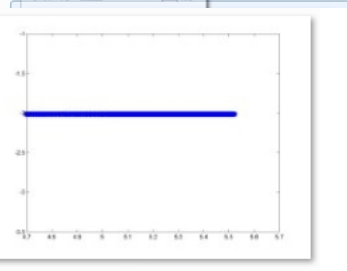

40.jpeg

Fig. 8 Double logarithm graph in fractal dimension calculation process of sample $8 \#$

From Fig.8, it could be found that there was the longest multi-collinearity interval and the greatest linear relationship at the graph of $M=10$. As a result, the value of $M$ was finally chosen as 10 , and we could get the multicollinearity interval of $[\ln 115, \ln 170]$, the relevant fractal dimension of $\tau=4.929943$, correlation coefficient of $r=0.99488, F$ check of $F=10493.09$ at this moment.

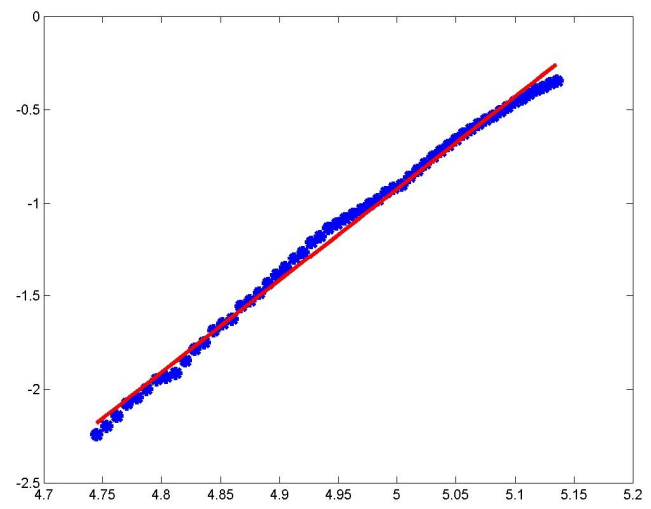

Fig. 9 The image $\ln N_{r}-\ln r$ of sample 8\#

After other samples are calculated in turn, it can obtain other samples' relevant fractal dimension. Because of the sample, 6\# and 17\# appear the obvious deivation, these two samples would not be considered in the samples from 1 to 25. 
Table2: computing result by Matlab of the sample's fractal dimension

\begin{tabular}{|c|c|c|c|c|c|c|}
\hline No. & correlation dimension & correlation coefficient & F test & $\begin{array}{c}\text { the left end } \\
\text { point of } \\
\text { collinear } \\
\text { interval } \\
\text { end point } \\
\text { of } \\
\text { collinear } \\
\text { interval }\end{array}$ \\
\hline 1 & 4.947299 & & & & & \\
value \\
\hline 2 & 4.89034 & -25.8347 & 0.983243 & 5222.352 & 100 & 190 \\
\hline 3 & 4.878329 & -25.1822 & 0.97312 & 3258.154 & 142 & 233 \\
\hline 4 & 4.757039 & -25.6958 & 0.985463 & 4270.89 & 116 & 180 \\
\hline 5 & 4.757039 & -25.6958 & 0.985463 & 4270.89 & 116 & 180 \\
\hline 7 & 4.608492 & -23.5894 & 0.985249 & 3272.786 & 110 & 160 \\
\hline 8 & 4.929943 & -25.5729 & 0.99488 & 10493.09 & 115 & 170 \\
\hline 9 & 4.727042 & -24.0174 & 0.987239 & 3790.683 & 110 & 160 \\
\hline 10 & 4.950186 & -24.8776 & 0.961194 & 1213.702 & 110 & 160 \\
\hline 11 & 4.566212 & -25.5758 & 0.994307 & 8557.626 & 170 & 220 \\
\hline 12 & 4.659387 & -23.5738 & 0.994882 & 7580.857 & 110 & 150 \\
\hline 13 & 4.729212 & -26.216 & 0.984537 & 7576.822 & 150 & 270 \\
\hline 14 & 4.967157 & -28.3649 & 0.994809 & 12265.74 & 185 & 250 \\
\hline 15 & 4.853667 & -26.4582 & 0.995308 & 10819.56 & 150 & 202 \\
\hline 16 & 4.71113 & -25.161 & 0.99342 & 10417.5 & 110 & 180 \\
\hline 18 & 4.610327 & -23.4355 & 0.982878 & 3099.788 & 110 & 165 \\
\hline 19 & 4.955938 & -25.9399 & 0.993055 & 11296 & 110 & 190 \\
\hline 20 & 4.864455 & -27.0862 & 0.994337 & 19488.24 & 138 & 250 \\
\hline 21 & 4.581916 & -23.3921 & 0.994929 & 9025.814 & 110 & 157 \\
\hline 22 & 4.825633 & -23.7072 & 0.954546 & 693.0134 & 110 & 144 \\
\hline 23 & 4.848863 & -27.7876 & 0.971807 & 3584.862 & 155 & 260 \\
\hline 24 & 4.914154 & -27.0859 & 0.994713 & 12040.7 & 135 & 200 \\
\hline 25 & 4.960986 & -24.8125 & 0.980301 & 1592.411 & 110 & 143 \\
\hline
\end{tabular}

\section{Study of the diameter distribution and filter efficiency physical property of electrospun nanofibres}

As electrospun nanofiber diameter distribution is too complex and unordered, the paper adopts the fractal theory to conduct study. Because the diameter distribution of fiber is caused by the dynamical system, it takes the correlation dimension commonly used in the dynamical system into the consideration. 23 samples' correlation dimension sees table 4, they have a close relation with samples'resistance.

The linear function relationship between sample fractal and its filter efficiencies can be expressed as $y=-0.0026 x+4.8548$ through Matlab fitting, $F$ check: be known of $F_{0.995}(1,24)=9.95$ after checking table

\begin{tabular}{|c|c|c|c|c|c|}
\hline $\begin{array}{c}\text { Sources of } \\
\text { variance }\end{array}$ & Sum of squares & $\begin{array}{c}\text { degrees of } \\
\text { freedom }\end{array}$ & square & F & conspicuousness \\
\cline { 1 - 3 } regression & SSR & 1 & SSR/1 & \multirow{2}{*}{10.3705} & $\begin{array}{c}\text { Extremely } \\
\text { significant }\end{array}$ \\
\hline residual error & SSE & 22 & SSE/22 & & \\
\hline sum & Lxx & 23 & & & \\
\hline
\end{tabular}

Correlation coefficient check, table-checking $1 \%, \mathrm{n}-2=21$, critical value of 0.526 and $\mathrm{r}=10.3705$, suggesting a significant relation, as shown in figure 10 . 


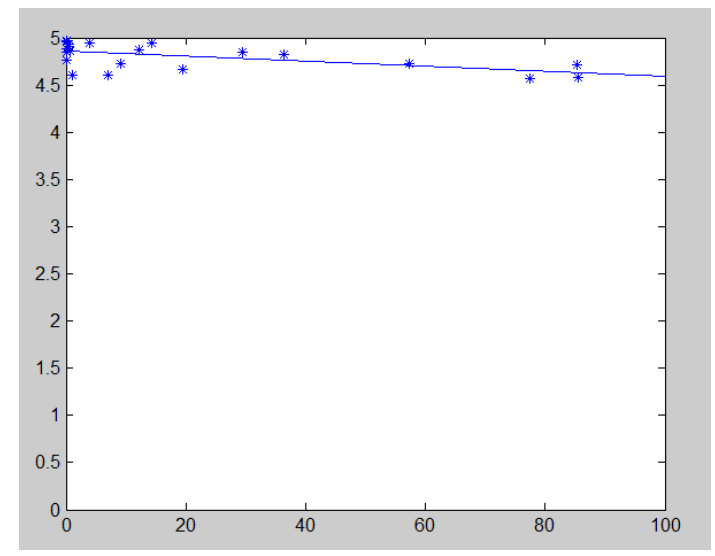

Fig. 10 Resistance of fractal image of quadratic function

Above all, we come out the conclusion that there is a linear relationship between the filtration efficiency of electrospun nanfiber nonwoven fabric and its relevant fractal dimension, namely when the higher the filter efficiency is, the smaller the fractal dimension is. This conclusion keeps the consistent with our usual thought, which also provides a new way to study the the properties of electrospun nanofiber nonwoven fabrics by fractual method.

Acknowledgements: We wish to thank the referees for their helpful comments and suggestions.

This research was supported by NSFC Grant No. 11071279, Foundation for the Author of National Excellent Doctoral Dissertation of China (201255), Program for New Century Excellent Talents in University (NCET-12-1063), Tianjin Natural Science Foundation (14JCYBJC17600)

\section{References}

[1] B. Christoph , K. J. Falconer, Fractals, Wavelets, and Their Applications: Contributions from the International Conference and Workshop on Fractals and Wavelets, Springer International Publishing AG,

[2] R. Chen, Wukui Zhao, Reseach of fractal of non-woven fabrics's filter and mechanical properties, Journal Of Tianjin Polytechnic University, 2009, Vol.28, No.3;30-33

[3] M. Fernández-Martínez, Sánchez-Granero, M.A. Fractal dimension for fractal structures, In Ibero-American Conference on Topology and its Applications (CITA-2012), Topology and its Applications 2014-02-15 163:93-111

[4] S. Zhang, Image Engineering(Second Edition), Tsinghua University Press, 2012.

[5] R. C. Gonzalez, Richard E. Woods, Steven L.Eddins, Digital Image Processing Using MATLAB (Second Edition), Electronic Industry Press, 2013.

[6] P. Grassberger \& I. Procaccia, [1983b] Measuring, the strangeness of strange attractors, Physica D9, 189-208.

[7] J. Serra, Introduction to Mathematical Morphology[M]. New York:Academic Press, 1982. 